\title{
A Smart Infrared Microcontroller-Based Blind Guidance System
}

\author{
Amjed S. Al-Fahoum, Heba B. Al-Hmoud, and Ausaila A. Al-Fraihat \\ Biomedical Systems and Informatics Engineering Department, Hijjawi Faculty for Engineering Technology, \\ Yarmouk University, Irbid 21163, Jordan \\ Correspondence should be addressed to Amjed S. Al-Fahoum; afahoum@yu.edu.jo
}

Received 20 March 2013; Revised 19 June 2013; Accepted 27 June 2013

Academic Editor: Liang Han

Copyright (C) 2013 Amjed S. Al-Fahoum et al. This is an open access article distributed under the Creative Commons Attribution License, which permits unrestricted use, distribution, and reproduction in any medium, provided the original work is properly cited.

\begin{abstract}
Blindness is a state of lacking the visual perception due to physiological or neurological factors. The partial blindness represents the lack of integration in the growth of the optic nerve or visual centre of the eye, and total blindness is the full absence of the visual light perception. In this work, a simple, cheap, friendly user, smart blind guidance system is designed and implemented to improve the mobility of both blind and visually impaired people in a specific area. The proposed work includes a wearable equipment consists of head hat and mini hand stick to help the blind person to navigate alone safely and to avoid any obstacles that may be encountered, whether fixed or mobile, to prevent any possible accident. The main component of this system is the infrared sensor which is used to scan a predetermined area around blind by emitting-reflecting waves. The reflected signals received from the barrier objects are used as inputs to PIC microcontroller. The microcontroller is then used to determine the direction and distance of the objects around the blind. It also controls the peripheral components that alert the user about obstacle's shape, material, and direction. The implemented system is cheap, fast, and easy to use and an innovative affordable solution to blind and visually impaired people in third world countries.
\end{abstract}

\section{Introduction}

Many people suffer from serious visual impairments preventing them from travelling independently. Accordingly, they need to use a wide range of tools and techniques to help them in their mobility. One of these techniques is orientation and mobility specialist who helps the visually impaired and blind people and trains them to move on their own independently and safely depending on their other remaining senses. Another method is the guide dogs which are trained specially to help the blind people on their movement by navigating around the obstacles to alert the person to change his/her way. However, this method has some limitations such as difficulty to understand the complex direction by these dogs, and they are only suitable for about five years. The cost of these trained dogs is very expensive, also it is difficult for many of blind and visually impaired persons to provide the necessary care for another living being. There is an international symbol tool of blind and visually impaired people just like the white cane with a red tip which is used to enhance the blind movement. Nowadays, different types of these canes have been used such as the white cane [1], the smart cane [2], and the laser cane [3]. However, this tool has several constraints: long length of the cane, limitations in recognizing obstacles, and also difficulty to keep it in public places. Recently, many techniques have been developed to enhance the mobility of blind people that rely on signal processing and sensor technology. These called electronic travel aid (ETA) devices help the blind to move freely in an environment regardless of its dynamic changes. According to the literature, ETAs are mainly classified into two major aspects: sonar input (laser signal, infrared signals, or ultrasonic signals) [4-8] and camera input systems (consists mainly of a mini CCD camera) [9-13]. Their details are shown in Table 1 . The way these devices operate just like the radar system that uses ultrasonic fascicle or laser to identify height, the direction, and speed of fixed and moving objects. The distance between the person and the obstacles is measured by the time of the wave travel. However, all existing systems inform the blind of the presence of an object at a specific distance in front of or near to him. These details permit the user to change his or her way. Information about the object characteristics can create additional knowledge 
TABLE 1: Existing wearable blind guidance systems.

\begin{tabular}{|c|c|c|c|c|c|c|c|c|}
\hline System type & System name & Reference & Year & Country & Signal input & Signal output & Advantages & Disadvantages \\
\hline \multirow{4}{*}{$\begin{array}{l}\text { Sonar } \\
\text { systems }\end{array}$} & NavBelt & {$[5]$} & 1998 & USA & Ultrasonic & $\begin{array}{c}\text { Auditory } \\
\text { display }\end{array}$ & \multirow{4}{*}{$\begin{array}{l}\text { Can provide range of } \\
\text { information of the obstacle }\end{array}$} & \multirow{4}{*}{$\begin{array}{l}\text { Cannot provide } \\
\text { precise information } \\
\text { such as shape and } \\
\text { motion state of } \\
\text { obstacles, and also } \\
\text { cannot make } \\
\text { judgments to the } \\
\text { travel orientation }\end{array}$} \\
\hline & People sensor & {$[6]$} & 1998 & USA & Ultrasonic & $\begin{array}{l}\text { Vibrotactile } \\
\text { cues }\end{array}$ & & \\
\hline & $\begin{array}{l}\text { 3D space } \\
\text { perceptor }\end{array}$ & [7] & 2003 & Canada & Laser & $\begin{array}{c}\text { Auditory } \\
\text { display }\end{array}$ & & \\
\hline & $\begin{array}{l}\text { Navigation } \\
\text { system based } \\
\text { on optical } \\
\text { peacans }\end{array}$ & {$[8]$} & 2001 & Japan & $\begin{array}{l}\text { Infrared } \\
\text { signal }\end{array}$ & $\begin{array}{l}\text { Auditory } \\
\text { display }\end{array}$ & & \\
\hline \multirow{5}{*}{$\begin{array}{l}\text { Camera } \\
\text { based } \\
\text { systems }\end{array}$} & v0ICe & [9] & 1992 & Netherlands & $\begin{array}{l}\text { CCD } \\
\text { camera }\end{array}$ & $\begin{array}{l}\text { Auditory } \\
\text { display }\end{array}$ & \multirow{5}{*}{$\begin{array}{l}\text { It provides information not } \\
\text { only about obstacles along } \\
\text { the traveled path } \\
\text { Assists the user in selecting } \\
\text { the preferred travel path } \\
\text { It can capture whole } \\
\text { environment information } \\
\text { It can use advanced image } \\
\text { processing algorithm to } \\
\text { train complex data }\end{array}$} & \multirow{5}{*}{$\begin{array}{l}\text { larger size, higher } \\
\text { cost relatively, and it } \\
\text { need two cameras so } \\
\text { as distance detection } \\
\text { algorithms is more } \\
\text { complex than } \\
\text { sonar-based ETAs }\end{array}$} \\
\hline & Tyflos & [10] & 2001 & USA & $\begin{array}{l}\text { Camera and } \\
\text { laser } \\
\text { scanner }\end{array}$ & $\begin{array}{l}\text { Auditory } \\
\text { display }\end{array}$ & & \\
\hline & NAVI & {$[11]$} & 2007 & Malaysia & $\begin{array}{c}\text { CCD } \\
\text { camera }\end{array}$ & $\begin{array}{l}\text { Auditory } \\
\text { display }\end{array}$ & & \\
\hline & AudioMan & [12] & 2007 & China & $\begin{array}{c}\text { CCD } \\
\text { camera }\end{array}$ & $\begin{array}{l}\text { Auditory } \\
\text { display }\end{array}$ & & \\
\hline & SoundView & [13] & 2009 & China & $\begin{array}{c}\text { CCD } \\
\text { camera }\end{array}$ & $\begin{array}{l}\text { Auditory } \\
\text { display }\end{array}$ & & \\
\hline
\end{tabular}

to enhance space manifestation and memory of the blind. To overcome the above-mentioned limitations, this work offers a simple, efficient, configurable electronic guidance system for the blind and visually impaired persons to help them in their mobility regardless of where they are, outdoor or indoor. The originality of the proposed system is that it utilizes an embedded vision system of three simple IR sensors and brings together all reflective signals in order to codify an obstacle through PIC microcontroller. Hence, in addition to distance the proposed guidance system enables the determination of two main characteristics of the obstacle which are material and shape. Furthermore, the user of the system does not need to carry a cane or other marked tool. $\mathrm{He} / \mathrm{she}$ can just wear a hat and hand mini stick (size of a pen) just like others. It has high immunity to ambient light and colour of object. It has typical response time about $39 \mathrm{~ms}$, and it is very suitable for real-time applications.

\section{Materials and Methods}

2.1. Block Diagram of the Proposed Smart MicrocontrollerBased Blind Guidance System. Proteus is software for microprocessor simulation, schematic capture, and printed circuit board (PCB) design. Proteus-VSM (Virtual System Modelling) permits the cosimulation of the embedded software for popular microcontrollers alongside with the hardware design [14]. The software Proteus was used to design and analyze both preliminary and final results for each part of the system [14]. Proteus enabled the system to be tested and modified until accurate and expected results were obtained prior to the circuit installation in practice. Figure 1 shows the design and the implementation of the proposed smart blind guidance system. The block diagram of the system is

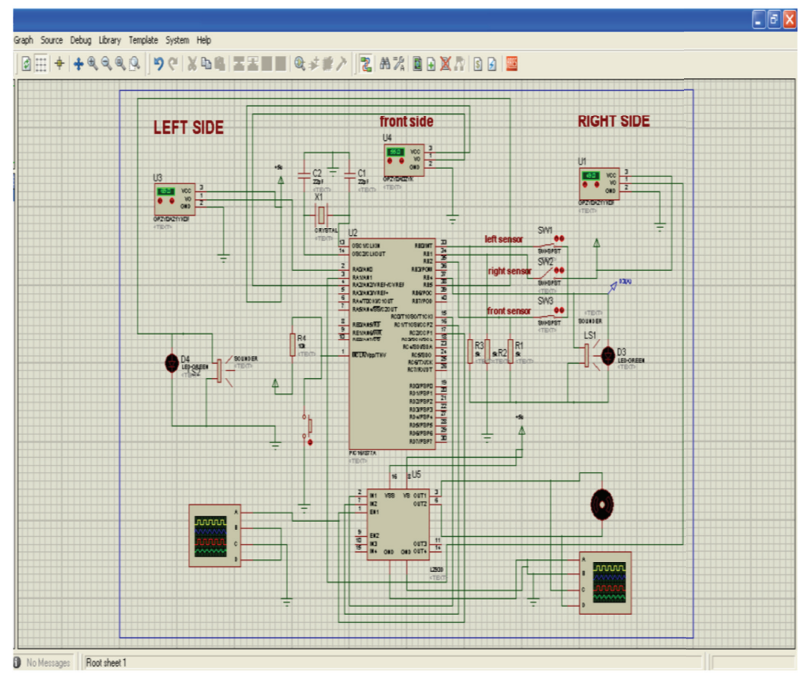

FIgUre 1: The whole circuit design using Proteus simulation.

illustrated in Figure 2. It shows three reflective signals that were produced as follow: from front IR sensor, right IR sensor, and from left IR sensor. All signals are inputs for ADC on a PIC microcontroller. These signals are digitized and used as inputs to a specific program implemented in real time within PIC microcontroller. As a result of these signals the microcontroller according to some internal instructions will produce an output which will be transferred from the PIC to the buzzer and motor driver IC to aware the blind about the barriers blocking his/her way.

2.1.1. PIC Microcontroller 16F877A. To make wearable obstacle detection system for visually impaired people respond 


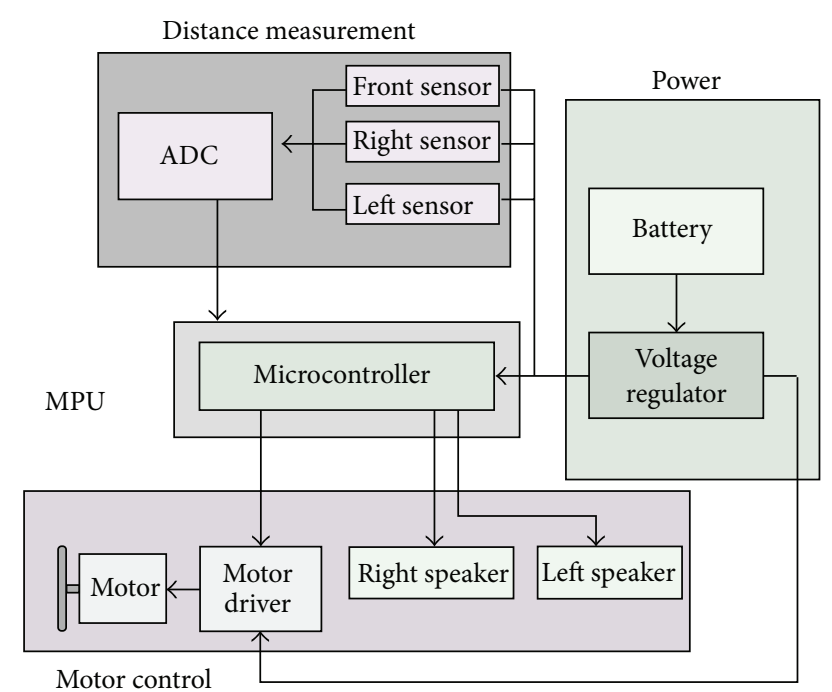

FIGURE 2: Block diagram of the system.

faster, it should be equipped with advanced microcontroller to decrease computational complexity. PIC 16F877A was chosen to detect any switch triggered and generate the audio sounds and vibrations. The PIC does not have an operating system and simply runs the program in its memory when it is turned on. PIC microcontroller is a small computer on a single integrated circuit which stores a set of instructions. It consists of a processor core, memory, and programmable input/output peripherals. PIC is an important component in the proposed system which deals with a MicroC programming code which was installed in it. The system is featured by its small size and low cost when it is compared with other systems that use separate microprocessor, input/output devices, and memory. Mixed signal microcontrollers are common, integrating analog components needed to control nondigital electronic systems. PIC microcontroller operates at $+5 \mathrm{~V}$ which can be regulated using the voltage regulator (L7805) which conserves voltage at $+5 \mathrm{~V}$ if the input voltage for it exceeds $+5 \mathrm{~V}$. Also PIC cannot run without using its crystal oscillator which is used to execute the programming code. The PIC is used as a real-time processing element; therefore, a high frequency oscillator is used [15].

2.1.2. IR Sensors. Three IR sensors are employed to acquire particular details relative to the obstacle categorization. In this paper, the suggested guidance system principle is shown by introducing the main sensors and their ability to detect and identify typical objects. The IR sensors are the main electronic components in the proposed system because it acts as the new eyes for the blind. One IR sensor is located on the hand mini stick to scan the front side, and the other two sensors are located on the hat at both right and left sides. IR sensors will scan all area in there range of IR beam. Any obstacle lies in the scanning range of the IR beam will be reflected and picked back by the receiver unit in the sensor. The distance and angle detection depend on the body that caused beam reflection. Figure 3 shows the block diagram of IR sensor.

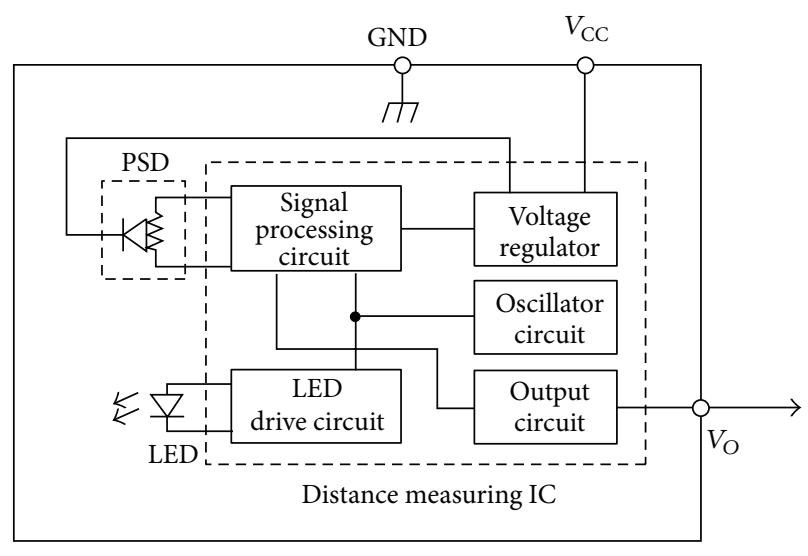

FIGURE 3: The block diagram of IR Sensor.

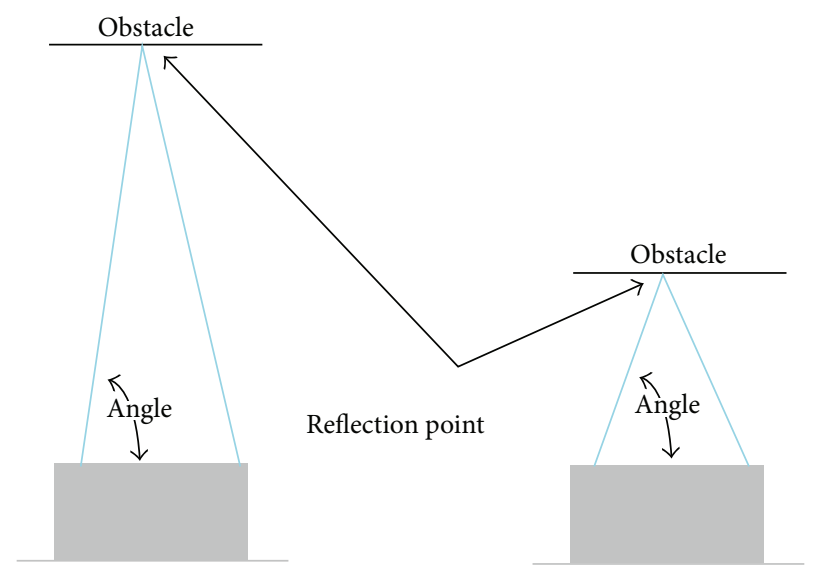

FIGURE 4: Different angles with different distances.

The range of distance to be scanned is controlled by a switch. Three switches are used to control the range of sensors in the three directions (i.e., when switches are on, the sensors will work at full range; otherwise they will work at their half range). The IR sensors are used to detect an obstacle besides (right and left) and in front of the blind at a distance between 10 and $150 \mathrm{~cm}$.

The basic tools in electronic IR guide are mini hand stick and head hat. These tools have IR sensors on it. These sensors represent the blind eyes so its position is very important to give right decision that depends on their output. Mini hand stick contains IR sensor with a distance range from 10 to $150 \mathrm{~cm}$. This range can be controlled by the PIC microcontroller, so it can be operated at half range $(75 \mathrm{~cm})$ via open switch button. What makes the sensor favourable is its small weight which is about $65 \mathrm{gm}$, also it offers proper protection for the sensor via thin transparent layer of plastic. The head hat is the second tool in the proposed blind electronic guidance system. The hat provides the system the ability to scan areas in the right and left side of the blind via two sensors fixed on it. The two sensors can work either on their full range $150 \mathrm{~cm}$ or their half range $(75 \mathrm{~cm})$ according to what is preferable by the user who can control it via switches. 
Theory of IR Sensor Operation. The new IR sensors use a developed approach that not only gives object detection at a longer range, but also offers a range of information. These new domains provide a much better immunity to ambient lighting conditions because of the new method of ranging. These new domains use a small linear CCD array and triangulation to calculate the distance and presence of objects in the field of vision. A pulse of infrared light is released by the emitter. This light travels across the specific area, if there is no obstacles in it, then the light never reflects, and the result shows no object, but if there is an obstacle in their way, then the light hits it, and it will be reflected. This action creates a triangle between the reflection point, the emitter, and the detector as shown in Figure 4. Triangle angles vary based on the distance of the object. The receiving part of these new detectors is in fact a precision lens that transmits the reflected light onto different parts of the enclosed linear CCD array according to the angle of the triangle described previously. The CCD array can determine at what angle the reflected light came back at, and therefore, it can calculate the distance to the object. This new method of ranging almost avoids the interference from ambient light and offers amazing in difference to the colour of object being detected. The infrared light is sent out from a transmitter to the object in front, by passing through a condense lens, so that the light intensity is focused on a certain point. Refraction occurs once the light hits the surface of the object. Part of the refracted light will be sent back to the receiver end, in which another lens will combine these lights and determine the point of impact. The light will then be passed on to an array of phototransistors. The position in which the light falls can be used to calculate the distance $(L)$ from the transmitter to the obstacle using the following formula:

$$
\frac{L}{A}=\frac{F}{X} \quad \text { Therefore } L=\frac{(F \times A)}{X} .
$$

Thus, the distance value from the phototransistors will be sent to the Signal Evaluation Module before it is changed to voltage, resulting in a change of voltage according to the measured distance as shown in Figure 5.

2.1.3. Reading IR Sensor Output with A/D Converter. The IR sensor's output voltages will change according to the detection distance. To calculate the approximate distance, the following formula is used:

$$
R=\left(\frac{2914}{[V+5]}\right)-1,
$$

where $R$ is distance in centimetre unit and $V$ is digital data from $A / D$ converter.

2.1.4. Speakers. Speakers are main indicators of the designed system through which the blind can easily determine the shape and material of surrounding bodies around him/her. There are two speakers: left and right. If the tune sound is due to the left speaker then there is an object in the range of left IR sensor, and when tune is due to the right, it means that an object in right side of blind exits. When both speakers

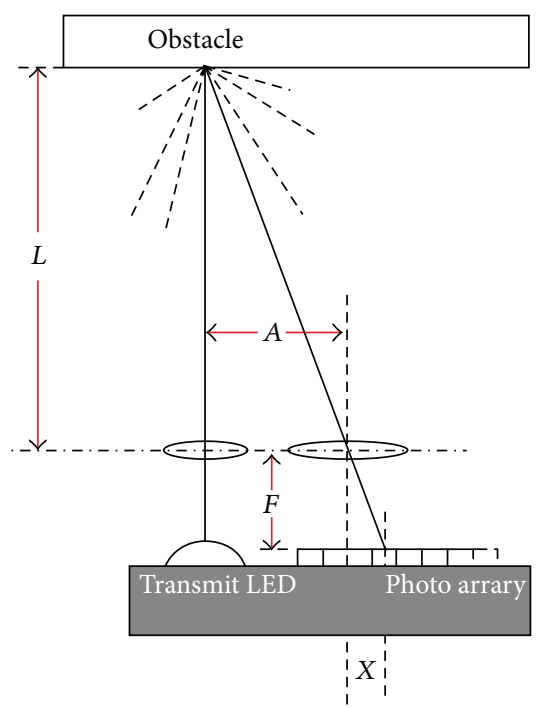

Figure 5: Distance measurement.

give the tune, it means that the obstacle is in front of blind. Another advantage for speakers is their small weight and size, so they do not constitute a burden to blind, and he/she can easily control the sound level via variable resistor to choose the sound level that does not make him/her annoyed.

2.1.5. Vibration Motor Driver. L293D is quad push-pull drivers designed to provide bidirectional drive current up to $1 \mathrm{~A}$ or $600 \mathrm{~mA}$ per channel. All channels are TTL-compatible logic inputs, and each output is a complete totem-pole drive circuit with Darlington transistor sink and pseudoDarlington source. The main function of L293D in this system is to control the current that is delivered to vibration motor using an enable pin that is connected directly to PIC. Vibration motor indicates how much the detected body in range is closer to blind in both left and right sides, and when the vibrator works with the left or the right speaker, then it indicates that there is a body in front and left or right according to speaker that was working. Figure 6 shows the signal that controls the motor and the incremental change in pulses width that result because of the decreased distance from the barrier.

2.1.6. Switches. The function of switches in this system is to control the range of the sensor. When switches are open (off), sensors operate at their half range, and when it is closed (ON), sensors operate at their full range.

\section{Results and Discussion}

The presented system is designed and configured for practical use. The system is able to handle seven states that may face the blind people. The system will respond to each state according to a specific program which is coded and installed in the PIC microcontroller. The first case is when the body is in the right direction of the blind, then just the right speaker will give sound. The second case is when the body is at the left side 


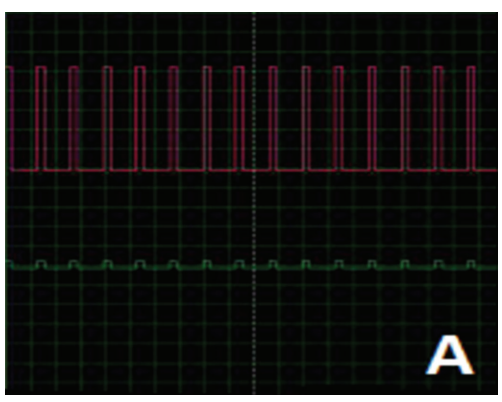

(a)

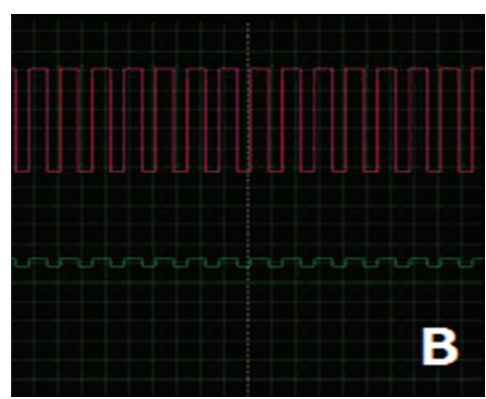

(b)

FIGURE 6: (a) Narrow ON time pulses (little vibration far distance), (b) wide $\mathrm{ON}$ time pulses (strong vibration close distance).

of the blind, then just the left speaker will give sound. The third case is when the body is in front of the blind, then both the left and right speakers will give sound. The forth case is when the body is located in the range of right and front sensors, that is, from front and right, then right speaker and vibration motor will work together. The fifth case is when the body is located in front and left sides of the blind, then left speaker and vibration motor will work. The sixth case is when the bodies are approaching the blind from both right and left directions, then just vibration motor will work. The last case is when the bodies come in all directions (front, right, and left), then both speakers and vibration motor will work (worst case scenario).

3.1. Trial Results. To distinguish an obstacle from another, it is crucial to acquire the key features of the objects, such as material and form. The obstacle categorization can sufficiently be differentiated by its characteristics, so that it can be detected. According to the main aim of this work and to make use of simple sensors, the infrared sensors have been examined to identify the obstacle materials and surfaces. All 10 male blind subjects, aged 18-50, participated in the test of the system. Every participant was taught to understand one path $(150 \mathrm{~m}$ long). The paths were selected to offer multiple obstacles of different materials of interest.

3.1.1. Materials Recognition. One of the main characteristics for obstacle different types to be detected is the differentiation by type of material as a first strategy. For four materials steel, glass, wood, and plastic, the emitted signal by the IR sensor was analyzed at a given distances. The microcontroller gathers
TABLE 2: Mean output voltage of the selected material.

\begin{tabular}{lc}
\hline Material type & Mean output voltage \\
\hline Wood & $0.928 \pm 0.025$ \\
Steel & $0.621 \pm 0.06$ \\
Plastic & $0.27 \pm 0.13$ \\
Glass & $0.75 \pm 0.03$ \\
\hline
\end{tabular}

TABLE 3: Results of shape recognition tests.

\begin{tabular}{lcc}
\hline Shape of object & Number of trials & Detection ratio \\
\hline Triangle pyramid & 10 & 93 \\
Cone & 10 & 93 \\
Cylinder & 10 & 96 \\
Rectangular pyramid & 10 & 94 \\
\hline
\end{tabular}

the reflected information from the obstacles by measuring the width of the echo pulse signal. The pulse width is directly pro portional to the distance of the nearest obstacle. By capturing the value of an internal counter 16 bits at rise and fall time of the reflected pulse, the microcontroller gets a 16 bit value of the distance. Obstacles were located successively from 10 to $150 \mathrm{~cm}$ (in the range of $10 \mathrm{~cm}$ ). The voltage mean values according to the four selected materials are summarized in Table 2 .

All the obtained data of the conducted trials were analyzed using SPSS. One way ANOVA followed by Turkey was applied with significance level of 0.05 . An important impact on the substance was noticed around the voltage $(F(1.25)=$ 55.7; $P<0,05)$. This result indicates that, using the infrared sensor, it can help to make a distinction between the four chosen material types.

However, the output signal of an infrared sensor depends on the material and the distance of the object. The longer the distance of the object, the smaller the output signal. To test the effect on these two factors, the voltage mean value according to the distance and material categories is illustrated in Figure 7. A significant effect of the voltage on the material according to the distance $(F(112)=77.8 ; P<0,05)$ was noticed. From the figure, it can be clearly seen that wood can be significantly differentiated from other materials at any distance. Regarding the other three materials, plastic, metal, and glass, material differentiation depends on the distance. At a distance less than $57 \mathrm{~cm}$ plastic can easily be detected. However, the system can differentiate between steel and glass at distances more than $57 \mathrm{~cm}$ from the head of the hand mini stick. Moreover, the system failed to make any discrimination of materials at distances more than $140 \mathrm{~cm}$ and less than $20 \mathrm{~cm}$.

3.1.2. Shape Analysis. In order to reduce material ambiguity according to the object distance (metal versus glass or plastic versus metal), a second IR sensor can be used to extract other object parameters, such as their shapes. The object form was extracted through voltage variation, while the object is scanned with the IR sensor in the hand mini stick. Table 3 shows that the system was successful in detection of 4 types 
TABLE 4: Comparison of different devices.

\begin{tabular}{lcccccc}
\hline System & Power consumption & Range & Portability & Invasiveness & Response time & Ease of usage \\
\hline The current suggested system & Low & Medium & Yes & Noninvasive & Fast & Friendly (no need for training) \\
NavBelt & Medium & Low & No & Noninvasive & Medium & Not friendly (needs training) \\
The white cane & High & Medium & No & Invasive & Medium & Not friendly (needs training) \\
NAVI & High & High & No & Noninvasive & Slow & Not friendly (needs training) \\
\hline
\end{tabular}

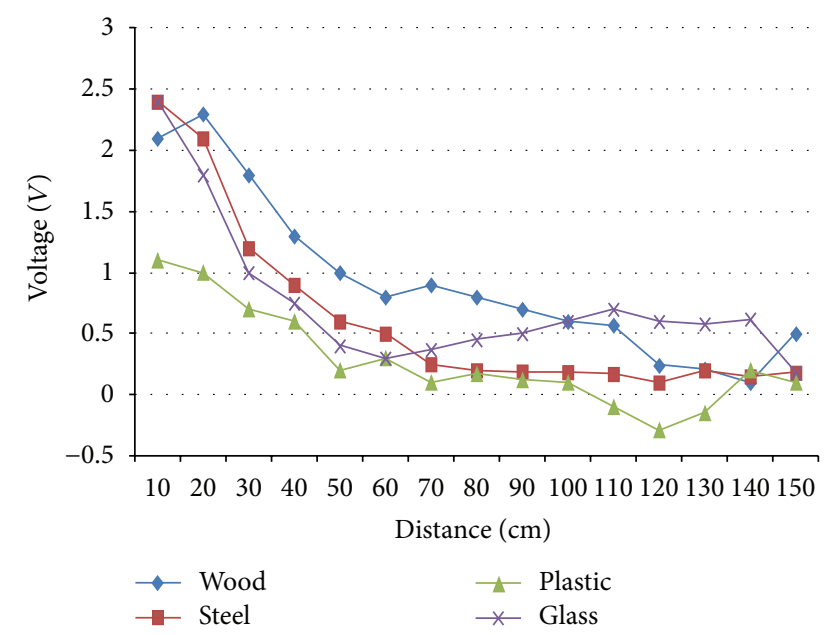

FIGURE 7: Voltage output at the object distance of different material categories.

of 3D different shapes (triangle pyramid, cone, cylinder, and rectangular pyramid). Therefore, the proposed system can be utilized for industrial applications.

3.1.3. System's Overall Accuracy. The system was tested on 10 blind subjects using obstacles of different shapes and materials at different distances. The results of the experiment are very encouraging. It revealed an accuracy of $93 \%$ which indicates that the system is efficient and unique in its capability in specifying the source and distance of the objects that may encounter the blind.

3.2. Parameters Specifications. To evaluate the performance of the proposed guidance system, several parameters should be considered. The first important parameter is the power consumption of the system and how long it will stay working without the need to recharge. The following ratings are considered: consumption of an electrical power of $0-0.5 \mathrm{~W}$ is regarded as low power, $0.5-1 \mathrm{~W}$ as medium consumption, and higher than $1 \mathrm{~W}$ as high consumption. The second parameter is the range of operation. A tool which can find obstacles throughout $0-2 \mathrm{~m}$ can be considered as a low range device, $2-4 \mathrm{~m}$ as medium range, while higher than $4 \mathrm{~m}$ is considered as high range. The third parameter is the response time, and a system sensing and responding $0-100 \mathrm{~ms}$ is regarded as fast, 100-200 ms medium and higher than $200 \mathrm{~ms}$ as slow. The portability is another important parameter of the system.
The system which can be worn and used by the subject for prolonged time is considered as a portable system; otherwise it is regarded as nonportable. The easiness of the system usage is considered as another parameter. An easy to use device is actually easy to get to and an easy to function. Finally the noninvasive utilization of the system is considered as a property of the system. Table 4 compares the aforementioned parametric specifications for different systems.

3.3. Comparisons with Other Systems. As mentioned in the introduction, any new technology should be compared with what is already available and up to date technology. The proposed system is compared with the NavBelt system which is worn by the user like a belt around the waistline and through a set of stereo earphones to provide acoustic signals which direct the user around obstacles. However, the NavBelt system suffers from certain limitations such as the difficulty of conveying information to the user to allow rapid walking, on the other hand the NavBelt must be used together with white cane $[4,16]$. At the stage of current development, it is clear that the white cane is potential in terms of cost, weight, electrical power, and reliability to reveal obstacles on the floor. However, if the floor is a fully reflective surface, then the cane laser scanning device fails. In addition to the revealed difficulties of the white cane in detecting hidden obstacles, it exhibits difficulty to the user in storing it in a public place $[16,17]$. The results show that the suggested system can detect more distant obstacles by using infrared and other sensing devices and can provide more precise range information of the obstacle than the aforementioned sonar systems. Moreover, the system overcomes the limitations of the camera-based system due to its noninvasive nature, low power consumption, cheap cost, simplicity, and ease of customization. However, even at this early stage of the system's implementation, there are many advantages in using the newly proposed system: first of all it is safe, inexpensive and can be worn everywhere; when there are some obstacles, the system alerts the user and allows him/her to make better understanding of the space around him/her due to the detection of the material and shape of the object. Hence, the blind can change his/her way and move more safely and easily. Although the proposed system was able to work successfully, there is some future works which will be focused on replacing the speaker's tune by real human sound to guide the blind exactly. Moreover, shape detection test for objects that move at different rotational speeds across several distances will further be considered. 


\section{Conclusion}

A simple, cheap, configurable, easy to handle electronic guidance system is proposed to provide constructive assistant and support for blind and visually impaired persons. The system is designed, implemented, tested, and verified. The real-time results of the system are encouraging; it revealed an accuracy of $93 \%$ in detecting different shapes, materials, and distances. The results indicate that the system is efficient and unique in its capability in specifying the source and distance of the objects that may encounter the blind. It is able to scan areas left, right, and in front of the blind person regardless of its height or depth. Therefore, it was favoured by those who participated in the test. The IR sensor has been fully utilized in order to advance the mobility of the blind and visual impaired people in safe and independent way. This system does not require a huge device to be hold for a long distance, and it also does not require any special training. This system also resolves limitations that are related to the most of the movement problems that may influence the blind people in their environment. Future work will be focused on enhancing the performance of the system and reducing the load on the user by replacing the speaker's tune by real human sound to guide the blind exactly. Moreover, shape detection test for objects that move at different rotational speeds across several distances will further be considered.

\section{References}

[1] D. Yuan and R. Manduchi, "Dynamic environment exploration using a Virtual White Cane," in Proceedings of the IEEE Computer Society Conference on Computer Vision and Pattern Recognition (CVPR '05), pp. 243-249, IEEE, San Diego, Calif, USA, June 2005.

[2] A. A. Tahat, "A wireless ranging system for the blind long-cane utilizing a smart-phone," in Proceedings of the 10th International Conference on Telecommunications (ConTEL '09), pp. 111-117, IEEE, Zagreb, Croatia, June 2009.

[3] D. Bolgiano and E. Meeks Jr., "A laser cane for the blind," IEEE Journal of Quantum Electronics, vol. 3, no. 6, p. 268, 1967.

[4] S. Shoval, I. Ulrich, and J. Borenstein, "NavBelt and the guidecane [obstacle-avoidance systems for the blind and visually impaired]," IEEE Robotics and Automation Magazine, vol. 10, no. 1, pp. 9-20, 2003.

[5] S. Shoval, J. Borenstein, and Y. Koren, "Auditory guidance with the navbelt-a computerized travel aid for the blind," IEEE Transactions on Systems, Man and Cybernetics C, vol. 28, no. 3, pp. 459-467, 1998.

[6] S. Ram and J. Sharf, "The people sensor: a mobility aid for the visually impaired," in Proceedings of the 1998 Digest of Papers $2 n d$ International Symposium on Wearable Computers, pp. 166-167, IEEE, October 1998.

[7] E. Milios, B. Kapralos, A. Kopinska, and S. Stergiopoulos, "Sonification of range information for 3-D space perception," IEEE Transactions on Neural Systems and Rehabilitation Engineering, vol. 11, no. 4, pp. 416-421, 2003.

[8] K. Magatani, K. Sawa, and K. Yanashima, "Development of the navigation system for the visually impaired by using optical beacons," in Proceedings of the 23rd Annual International Conference of the IEEE Engineering in Medicine and Biology Society, pp. 1488-1490, IEEE, October 2001.
[9] P. B. L. Meijer, "An experimental system for auditory image representations," IEEE Transactions on Biomedical Engineering, vol. 39, no. 2, pp. 112-121, 1992.

[10] N. G. Bourbakis and D. Kavraki, "An intelligent assistant for navigation of visually impaired people," in Proceedings of the 2001 IEEE 2nd International Symposium on Bioinformatics and Bioengineering Conference, pp. 230-235, IEEE, 2001.

[11] G. Sainarayanan, R. Nagarajan, and S. Yaacob, "Fuzzy image processing scheme for autonomous navigation of human blind," Applied Soft Computing Journal, vol. 7, no. 1, pp. 257-264, 2007.

[12] Z.-G. Fang, J. Xu, F.-l. Bao, and L.-H. Zhang, "AudioMan: design and implementation of environmental information data mapping," Chinese Journal of Ergonomics, vol. 2, article 001, 2007.

[13] M. Nie, J. Ren, Z. Li et al., "SoundView: an auditory guidance system based on environment understanding for the visually impaired people," in Proceedings of the 31st Annual International Conference of the IEEE Engineering in Medicine and Biology Society: Engineering the Future of Biomedicine (EMBC '09), pp. 7240-7243, IEEE, September 2009.

[14] B. Su and L. Wang, "Application of Proteus Virtual System Modelling (VSM) in teaching of microcontroller," in Proceedings of the International Conference on E-Health Networking, Digital Ecosystems and Technologies (EDT '10), pp. 375-378, IEEE, April 2010.

[15] J. Burroughs, "X-10 home automation using the PIC16F877A," Lamp, vol. 10, article 10, 2010.

[16] D. Dakopoulos and N. G. Bourbakis, "Wearable obstacle avoidance electronic travel aids for blind: a survey," IEEE Transactions on Systems, Man and Cybernetics C, vol. 40, no. 1, pp. 25-35, 2010.

[17] S. Chumkamon, P. Tuvaphanthaphiphat, and P. Keeratiwintakorn, "A blind navigation system using RFID for indoor environments," in Proceedings of the 5th International Conference on Electrical Engineering/Electronics, Computer, Telecommunications and Information Technology (ECTI-CON '08), pp. 765768, IEEE, May 2008. 

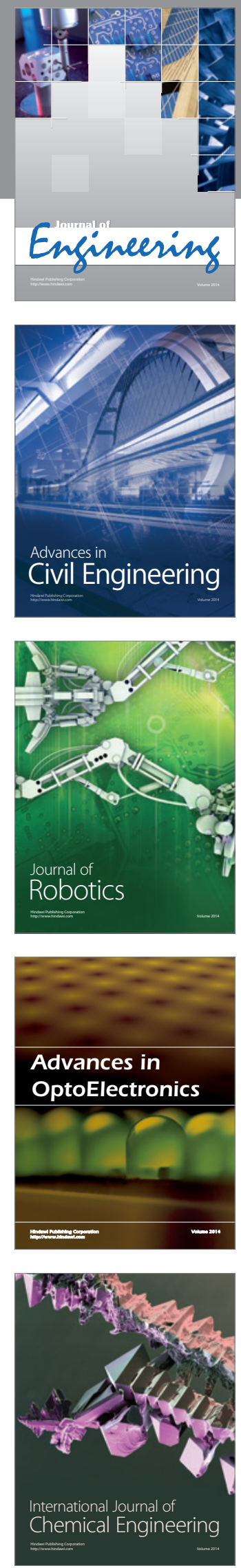

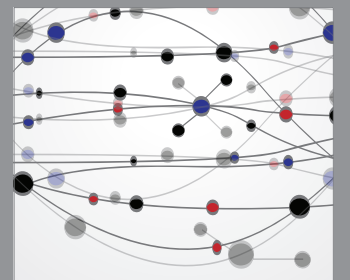

The Scientific World Journal
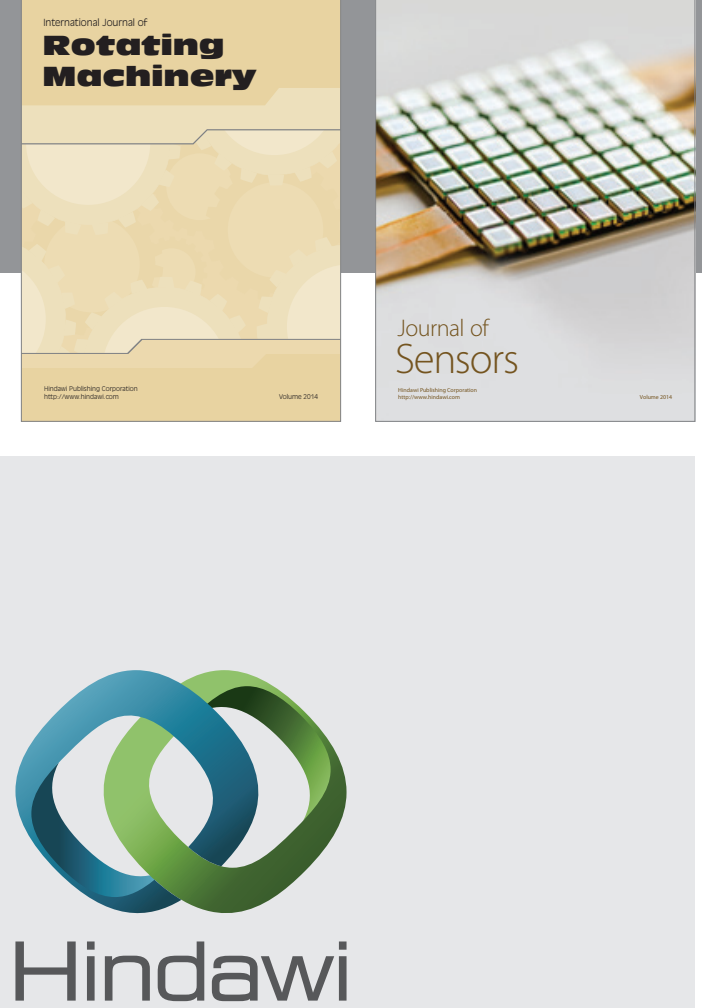

Submit your manuscripts at http://www.hindawi.com
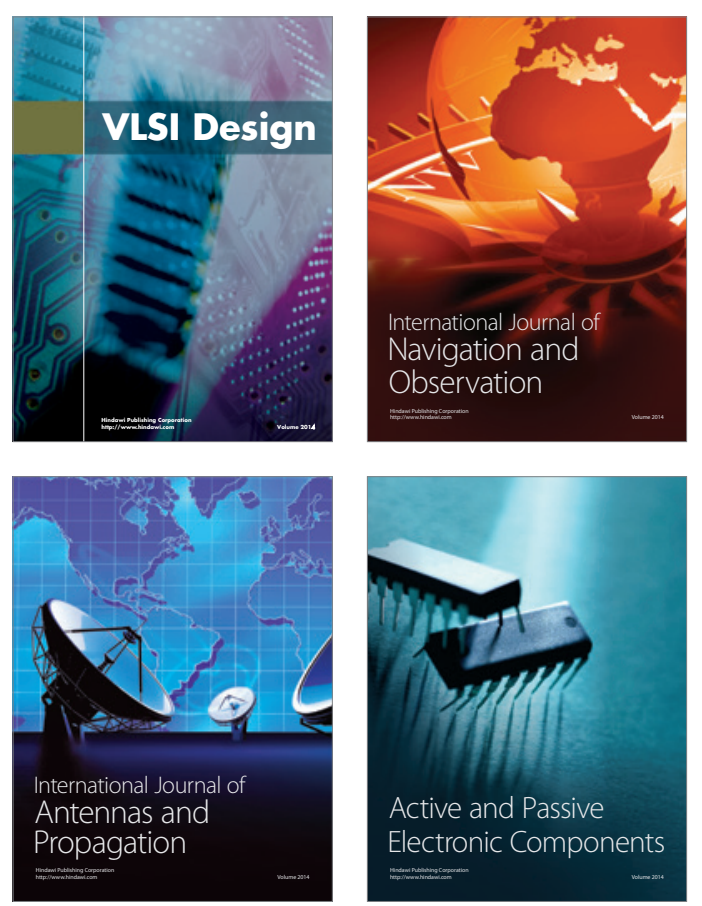
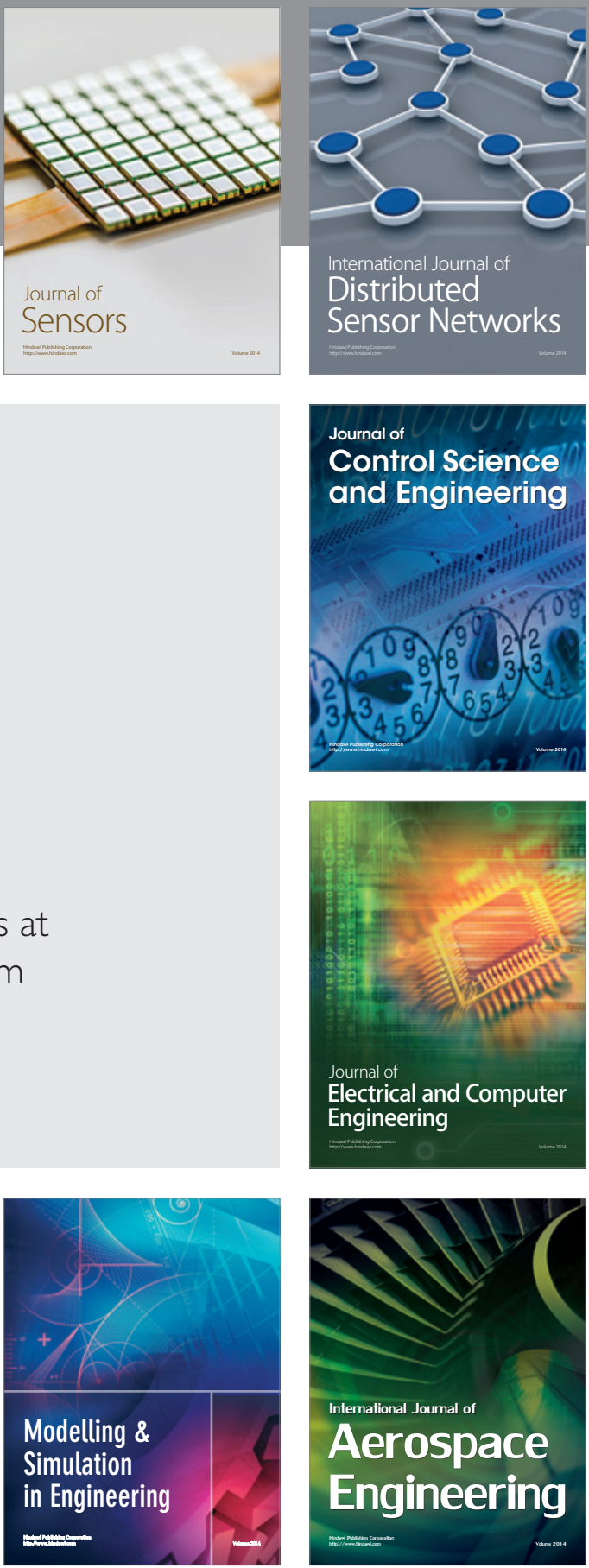

Journal of

Control Science

and Engineering
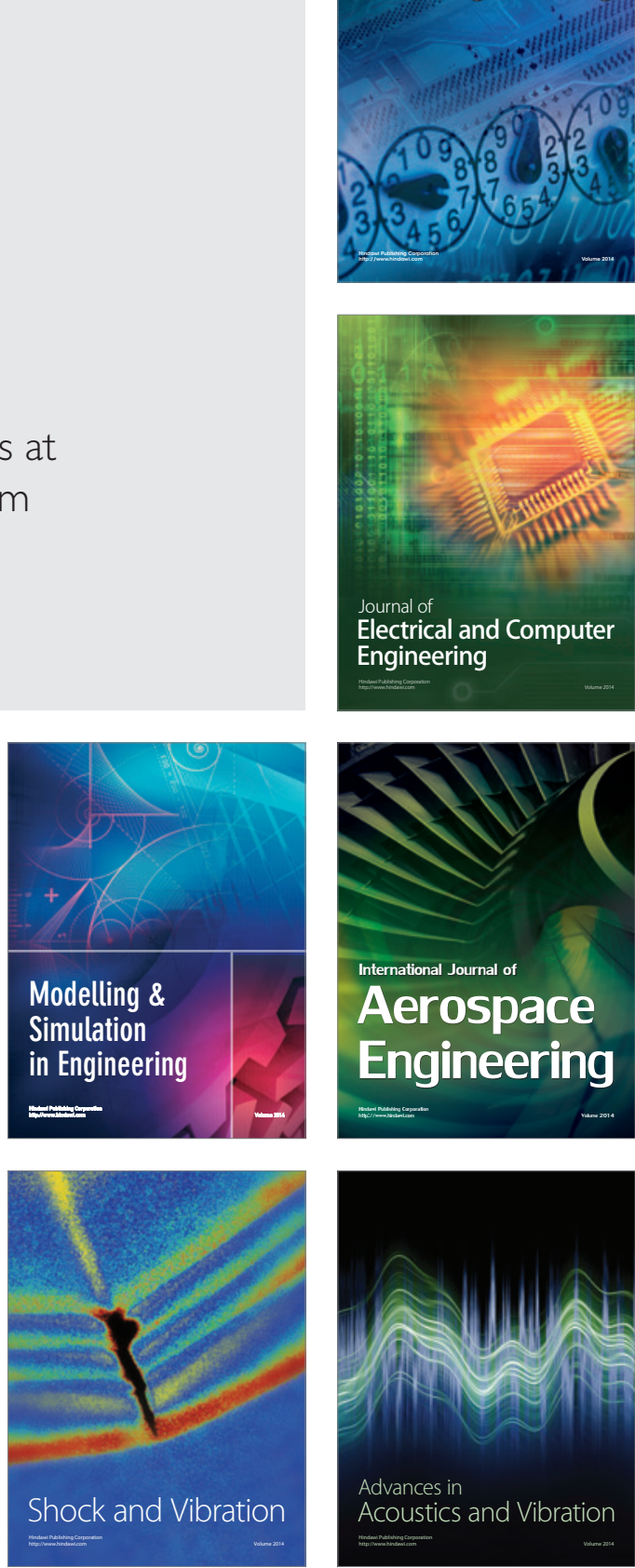